\title{
IV. - ERRATA
}

\section{au Bulletin géodésique n॰ 26,}

avril-mai-juin $r_{930}$.

\section{NOTES DE WALTER D. LAMBERT}

An approximate Rule for the Distance between the Geoid and the Spheroid on the Assumption of complete isostalic Compensalion of the Topography.

Page 9ı, ligne 2. Au lieu de Senior, Mathematician U. S, Coasl and Geodetic Survey, lire Senior Malhematician, U. S. Coast and Geodetic Survey.

The Form of the Geoid on the Hypothesis of complete isostatic Compensation.

Page 98, ligne 2. Au lieu de Senior, Mathematician, U. S. Coast and Geodetic Survey, lire Senior Mathematician, U. S. Coast and Geodetic Survey.

The Reduction of observed Values of Gravily to Sea Level.

Page ${ }_{10}$, ligne 2. Même correction que la précédente.

Page 122 , ligne 5. Au lieu de speroid, lire spheroid.

Page 123 , ligne 13 . Au lieu de preceeding, lire preceding.

Page 130 , ligne 5. Au lieu de $+0,0000000021 \cos 2 \varphi$, lire $-0.0000000021 \cos 20$.

Page 142 , lignes 8 et 9 en remontant. Remplacer le point et virgule par une virgule.

Page 148 , ligne 12 en remontant. Au lieu de applies, lire apply.

Page I 49, ligne 4. Au lieu de $\mathrm{AB}$, lire $\mathrm{OB}$.

Page 155 , ligne 7 (formule $\mathrm{B}_{n}=$ ). Au lieu de $\mathrm{P}_{43}$, lire $\mathrm{P}_{n-3}^{\prime}$.

Page 159 , ligne 19 . Au lieu de avoid violence, lire avoid doing violence.

Page $\mathbf{6 9}$, ligne $\mathbf{~}$. Aulieu de $\frac{d}{a}$, lire $\frac{d}{2 a}$.

- ligne I3. Au lieu de I. 8, lire I. 8.

Page 177 , ligne 16 . Supprimer that.

Page 179 , ligne ro, Au lien de profandità, lire profundità.

Page I8o, ligne I4. Au lieu de Schwereduktion, lire Schwerereduktion.

- ligne I en remontant. Au lieu de his, lire is.

Toulouss. - Impr. et Libr. Ebodand PRrvat. $-2336 .-8-193$ I 